\title{
Behavioral Change Challenges in the Context of Center-Based Cardiac Rehabilitation: A Qualitative Study
}

\author{
Fariba Jokar ${ }^{1}$; Hojatollah Yousefi ${ }^{2, *} ;$ Alireza Yousefy $^{3}$; Masoumeh Sadeghi ${ }^{4}$ \\ ${ }^{1}$ Students Research Center, School of Nursing and Midwifery, Isfahan University of Medical Sciences, Isfahan, IR Iran \\ 2 Department of Adult Nursing, School of Nursing and Midwifery, Nursing and Midwifery Care Research Center, Isfahan University of Medical Sciences, Isfahan, IR Iran \\ 3 Department of Medical Education, Isfahan University of Medical Sciences, Isfahan, IR Iran \\ ${ }^{4}$ Cardiac Rehabilitation Research Center, Isfahan University of Medical Sciences, Isfahan, IR Iran \\ ${ }^{*}$ Corresponding Author: Hojatllah Yousefi, Department of Adult Nursing, School of Nursing and Midwifery, Nursing and Midwifery Care Research Center, Isfahan University of \\ Medical Sciences, Isfahan, IR Iran. Tel: +98-3137927543, Fax:+98-3136699398, E-mail:yousefi@nm.mui.ac.ir
}

Received: January 30, 2015; Revised:April 5, 2015; Accepted: April 18, 2015

\begin{abstract}
Background: Cardiovascular disease is chronic and often a sign of long-standing unhealthy lifestyle habits. Patients need support to reach lifestyle changes, well-being, join in social and vocational activity. Thus, patient responsibility should to be encouraged to provide quality as well as longevity. Cardiac rehabilitation programs aid patients in the attainment of these objectives. However, research shows that behavioral change following the diagnosis of a chronic disease is a challenge.

Objectives: We sought to determine behavioral change challenges in patients with cardiovascular disease to improve intervention programs.

Patients and Methods: Using a descriptive qualitative approach, we collected the data using 30 in-depth semi-structure interviews. Thematic analysis was conducted to identify themes from the data.

Results: Three sources of behavioral change challenges emerged regarding the nature of cardiac disease and the role of the individual and the family in the recovery process. These challenges acted at two levels: intra- and interpersonal. The intrapersonal factors comprised value, knowledge and judgment about cardiovascular disease, and self-efficacy to fulfill the rehabilitation task. Family overprotection constituted the principal component of the interpersonal level.

Conclusions: Behaviors are actually adopted and sustained by patients are so far from that recommended by health professionals. This gap could be reduced by identifying behavioral change challenges, rooted in the beliefs of the individual and the family. Therefore, culturally-based interventions to enhance disease self-management should be considered.
\end{abstract}

Keywords: Behavior; Rehabilitation; Analysis

\section{Background}

World Health Organization's projections for 2030 suggest about 51.5 million deaths (76\% of the global mortality), demanding a course of action based on improvement in lifestyle and health behaviors (1). Progress in the methods of the diagnosis and treatment of cardiovascular disorders has conferred increased survival. Cardiovascular disease is a chronic condition that often reflects long-term poorly lifestyles habits; therefore, health care systems in their endeavors to overcome this particular challenge require more than high-tech equipment and pharmacological interventions. Cardiac patients need particular attention to rebuild their life quality, restore or improve their functional capacity (2), and effect necessary lifestyle modifications to be able to resume their social and vocational role in society, all of which places great responsibility on the patient in the pursuit of enhanced quality of life and longevity. Thus, patient responsibility should to be encouraged to provide quality as well as longevity. Cardiac rehabilitation programs are a vehicle for achieving these goals (3). Human behavior plays an important role in health and disease. Therefore, behavioral interventions effectively improve disease management and quality of life. Behavioral interventions can also prevent disease and decrease health costs (4). Such interventions are vital to the effective performance of clinical medicine and community health and can be defined as a series of coordinated actions to change behavior (5). Psychological reactions to cardiovascular disease are different. The assessment of the prospects of recovery is one of the dimensions of psychological reactions (6). Health behavior models indicate that lifestyle changes after the diagnosis of a chronic disease may be difficult (7). These theories have not addressed the issue of behavioral change in the context of chronic diseases, however. The core tenets of health behavior theories propose that the diagnosis of a chronic disease should encourage lifestyle changes. A variety of social, cognitive, and emotional factors are associated with lifestyle changes (8), and psychological status is one of the most important factors that influence physical activity and social functioning (1). Societal costs in the absence of appropriate disease management

Copyright ( 2 2015, Iranian Red Crescent Medical Journal. This is an open-access article distributed under the terms of the Creative Commons Attribution-NonCommercial 4.0 International License (http://creativecommons.org/licenses/by-nc/4.0/) which permits copy and redistribute the material just in noncommercial usages, provided the original work is properly cited. 
increase the number of patients with chronic illnesses (9), which highlights the need for the development of behavioral and health care system interventions capable of facilitating lifestyle improvements among cardiac disease patients. The process of behavioral change comprises several steps, each of which presents its own challenges and demands that have to be mastered. Physical disabilities and a sense that "nothing helps" with heart disease progression are factors allied to unsuccessful behavioral change (10). Individuals' confidence in their ability to make changes to their lifestyle, together with their perception of barriers to these changes, can considerably impact their ability to adhere to the management plan (11). Accordingly, knowing patients' experience of behavioral change barriers can effectively help health care teams in the design of behavioral interventions.

\section{Objectives}

This study aimed to explore cardiovascular disease patients' experience of behavioral change in the context of a center-based cardiac rehabilitation program.

\section{Patients and Methods}

\subsection{Setting and Participants}

The present study was commenced in November 2012 through interviews with individuals participating in a cardiac rehabilitation program at the two cardiac rehabilitation centers of Isfahan University of Medical Sciences. The inclusion criteria were comprised of attending at least 4 sessions of cardiac rehabilitation, diagnosis of ischemic heart disease, interventional treatment, high blood pressure, history of coronary artery bypass graft surgery, willingness to participate in the study, and absence of cognitive and speech disorder. Deemed best suited to provide a comprehensive picture of the phenomenon under investigation, purposive sampling was utilized for participant selection (12). The sample size was determined based on the principle of saturation, a process by which researchers collect and analyze data until no new themes are emergent. Saturation may sometimes be reached with 12 to 20 participants (13), and our research group achieved data saturation after the analysis of 30 interviews. The demographic and situational characteristics of the study population are depicted in Table 1.

\subsection{Data Collection}

Using a descriptive qualitative approach, we collected data through in-depth interviews. Via open-ended questions asked during semi-structured interviews, the study explored the participants' comprehension of the challenges with which they were faced regarding behavioral change in a cardiac rehabilitation program.

The first author (F.J.) referred to the two cardiac rehabilitation centers and furnished the potential study par- ticipants with comprehensive explanations about the research. Those who provided signed informed consents were asked to be present for interviews at a certain time and place. The interviews lasted between 45 and $100 \mathrm{~min}-$ utes and were all digitally recorded. After the end of each interview, the interviewer noted the salient points during the interview. The interviews were transcribed verbatim.

Table 1. Participants' Demographic and Situational Characteristics

\begin{tabular}{lcc}
\hline Characteristics & Men & Women \\
\hline Age, $\mathbf{y}$ & $45 \pm 7$ & $56 \pm 9$ \\
\hline Education & & \\
\hline Illiterate & 1 & 1 \\
\hline Primary school & 4 & 1 \\
\hline High school & 10 & 3 \\
\hline University degree & 7 & 3 \\
\hline Treatment & & \\
\hline Coronary artery bypass graft surgery & 10 & 5 \\
\hline Stent & 7 & - \\
\hline Only drug therapy & 5 & 3 \\
\hline Cardiac rehabilitation sessions & & \\
\hline 4 - 6 & 8 & 1 \\
\hline 7 - 13 & 9 & 3 \\
\hline 14 - 20 & 5 & 4 \\
\hline
\end{tabular}

\subsection{Ethical Considerations}

This study was approved by the Review Board of Isfahan University of Medical Sciences (\# 391158). Participation was voluntary, and written informed consent form was signed by all the participants. The participants were reassured regarding confidentiality and anonymity. To preserve anonymity, all the audio data and transcripts were encoded. The study was conducted in accordance with the principles of ethical publishing.

\subsection{Data Analysis}

The version of a thematic analysis developed by Clarke (2006) provides a robust, systematic framework for coding qualitative data. So, thematic analysis was conducted to identify themes from the data (14). During data analysis, the authors adopted an inductive approach and independently read the transcripts, utilizing open coding techniques whereby the text was broken down and coded according to its meaning. The emergent categories and themes were compared and contrasted, and agreement was achieved through discussion between the researchers. The interview files were consulted if the responses or analyses required explanation. The process was recursive in nature, with the authors moving back and forth between the stages of data familiarization and concept development until the themes were agreed upon. 


\section{Results}

The main theme, namely behavioral change challenges, reflected the participants' experience regarding behavioral change obstacles during rehabilitation. The main theme, themes, sub-themes, and their relations are shown in Figure 1.

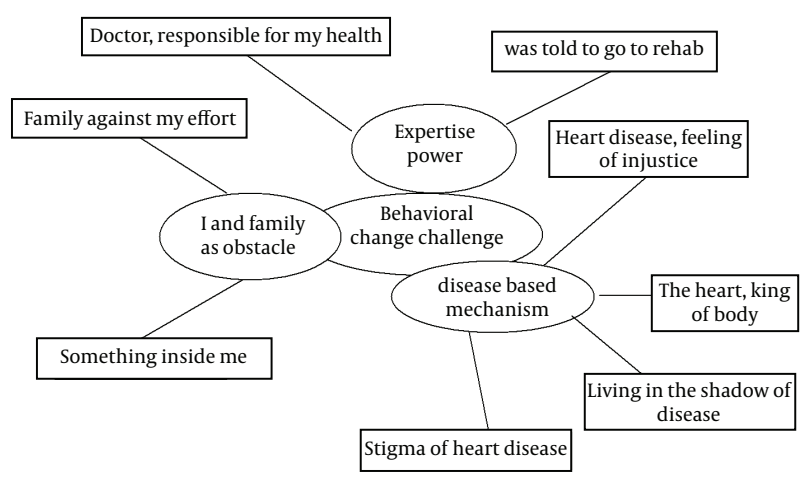

Figure 1. Behavioral Change Challenge Thematic Map

Three sources of behavioral change challenges include: 1) beliefs about the nature of cardiac disease; 2 ) the role of the individual and the family in the recovery process; and 3) responsibility for restoring health.

"Disease-based mechanism" is a theme discussing belief about the importance of the heart, impacts of it on life, and heart disease stigma. These beliefs have a deterrent effect on changing behavior during cardiac rehabilitation because individuals with chronic diseases usually redefine their identity in a new context. This theme emerged from the articulation of four sub-themes, namely heart disease, feeling of injustice; the heart, king of the body; living in the shadow of the disease; and stigma of heart disease. The participants expressed a sense of injustice vis-à-vis the diagnosis of cardiovascular disease, as is illustrated in the following excerpt from the interviews:

"What am I doing wrong? Why should I get involved with heart disease? I have not drunk alcohol. I am an innocent person." (male, 46 years old)

The participants viewed heart disease as a punishment for individuals doing wrong, hence the sense of injustice. The function of the heart and its role in the body caused the participants to regard heart disease as untreatable. This viewpoint can, therefore, have inhibitory effects on interventions, as is illustrated in the next paragraph:

"Eye, kidney, hand... diseases are curable with medication, but the diseased heart... no, no." (female, 59 years old) Also, the participants expressed the notion that cardiac disease is concomitant with imminent death and stated that it was the reason why they sought to conceal their illness from relatives and friends. The following sentence highlights the above-mentioned concept:

"I even told my sister I had a heart attack, even taking my medication stealthily." (male, 59 years old)
Keeping one's illness secret is, thus, a threat to patient compliance with the treatment plan.

The subjects remarked that cardiovascular disease had affected all aspects of their lives, changing not only their body but also their self-understanding, their job, their interaction with others, and their expectations until ultimately everything changed. Clearly, this is a situation far from desirable, as the following statement testifies:

"You get back pain, so you're not lifting heavy objects. Then your pain is relieved. But cardiac disease affects all your life, diet, activity, and work. It bothers me." (Female, 49 years old)

"The expert's power" is a theme dealing with the relationship between the patient and the physician and how this relationship is defined. The participants viewed physicians as an authority responsible for maintaining their health. They granted physicians this status because of their knowledge and skills, as is attested to by the following sentence:

"My doctor told me to participate in rehab, so I came." (male, 68 years old)

"I do not know what is good or bad for me, so my doctor should tell me and manage the disease problems." (female, 69 years old)

Almost all the participants pointed to the theme of "the expert's authority", with their acceptance of this superiority stemming from uncertainty in their own ability to manage the disease.

Another theme, "I and family as an obstacle", pointed to the issues concerning family overprotection and patients' mood changes, which lessened our subjects' active engagement in the rehabilitation sessions. The participants remarked that these factors caused them to lose trust in the strength of their heart, as the following sentence illustrates:

"They even bring me a glass of water to swallow pills, not allowing me to carry a small object." (female 60 years old)

One of our male participants, who was retired and had undergone coronary artery bypass graft surgery, discussed this issue by saying:

"A few months after surgery when I wanted to remove a small pot, my wife did not allow it."

Participant discussed the instability in their mood and expressed it as "impatience" and "I can't do "so this instability caused interruption their effort for gaining healthy heart as shown in the next statements:

"Sometimes, something inside me tells me not to go. For example, some days I did not have the energy to go to rehab." (female, 45 years old)

"I can't run on the treadmill. It is difficult to do it with a sick heart." (female, 68 years old)

Having one's motivation for achieving a healthy heart lifestyle undercut by one's own mood instability is another hurdle on route to desirable behavioral change.

Our subjects underscored their uncertainty about the functional capacity of their diseased heart and intro- 
duced it as one of the reasons behind their inadequate physical activity. The following sentence serves as an example of such an attitude:

"I told myself this is enough when I exercised on the machines. My body can do it, but my mind says, 'no, it's enough.' I try not to tire my heart too much." (male, 51 years old)

All the aforementioned themes are cognitive factor that can interrupt the process of behavior change so change behavior does not happen as the main consequence of cardiac rehabilitation.

\section{Discussion}

Behaviors are actually adopted and sustained by patients are so far from that recommended by health professionals. This gap could be bridged by identifying the challenges of behavioral change. The diagnosis of chronic diseases represents a potential "wake-up call”, a chance to make important lifestyle changes, so the patient moves back and forth between two opposite poles: the desire to change and the resistance to change in the context of cardiac rehabilitation. As is illustrated in Figure 2 , the variables regarding behavioral change challenges among our study population acted at intra- and interpersonal levels. The factors at intrapersonal level were disease-oriented (heart disease, feeling of injustice; the heart, king of the body; living in the shadow of the disease; and stigma of heart disease), while those at interpersonal level concerned the patients' and their relatives' attitude toward the patients' capabilities after heart disease.

A stigma is an attribute that extensively discredits an individual, decreasing his or her from a whole and usual person to a tainted, reduced one. Major et al. (15) (1998) suggested that stigmatization happens when an individual possesses (or is believed to possess) an attribute or characteristic that projects a social identity that is undervalued in a particular social framework. Stigmatizing symbols may be obvious or invisible, manageable or unmanageable, and linked to physical appearance and behavior.

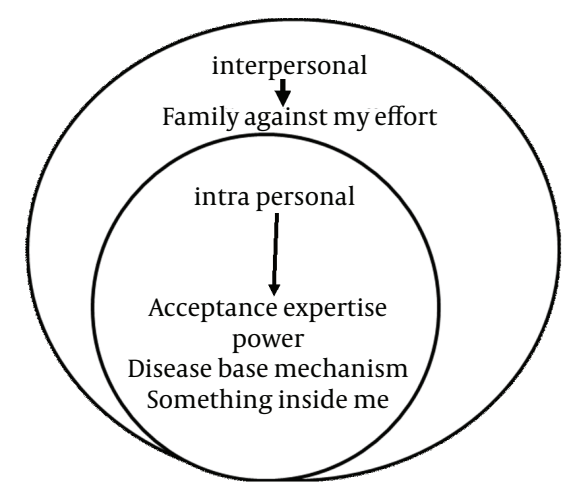

Figure 2. Behavioral Change Challenge Level
A self-stigma is defined as a phenomenon whereby an individual with a behavioral health condition internalizes a stereotype and applies it to herself or himself. Self-stigma interferes with help-seeking through three processes: 1) label avoidance; 2) diminished self-esteem; and 3) low self-efficacy (16). Our results showed that selfstigma impacted on the rehabilitation process through the participants' diminishing self-efficacy to begin behavioral change (Figure 1). The interpersonal variables that correlate with exercise among patients with cardiac disease include self-efficacy (e.g. one's belief in one's abilities to carry out an action). Self-efficacy is an important predictor of behavioral change (17). Cardiac patients' view on the importance of the heart in the body can be improved by providing correct information about their heart problem. The association between health knowledge deficit and failing health has been supported across acute and chronic diseases. Educational interventions should be literacy-sensitive and aimed at improving patients' health knowledge and self-efficacy as well as encouraging selfcare behavior and promoting health outcomes (18). Patients tend to draw upon their cognition and emotional beliefs regarding their illness to "make sense" of it. Such conceptualizations are formed based on beliefs about the chronology of the disease and its consequences, emotional response to the disease, beliefs about personal control over the illness and treatment efficiency, and the degree to which persons perceive sufficient understanding of the disease (19). Perception of an illness is not generally predicted by the clinical measures of illness severity, so there is no association between beliefs about the illness and the clinical markers of the illness (20). Thus, disease self-management behaviors are guided by patients' cognitive representation of the illness. Patients' beliefs about an illness and what it means with respect to their life are allied to their perception of the illness. The most important components include cause, timeline, consequences, symptoms, and cure or control. Patient models vary wildly across chronic conditions among individuals with the same disease severity (21). The relative impact of chronic diseases on the quality of life is diverse across similar conditions (22); thus, behavioral change challenges may vary because of the different perceptions of the illness as a threat to the quality of life or health. Cardiac self-management can often be ambiguous to many patients who have previously expected that their conditions would be solely managed by their physicians. "Disease-based mechanism" is a theme discussing disease-related beliefs that are liable to hinder behavioral change. That is why behavioral change interventions should be aimed at altering these beliefs. Patients' perception of their cardiovascular disease should be assessed during cardiac rehabilitation, and educational and psychosocial interventions should be devised to ensure that it does not act as a barrier to the behavioral change process. Cardiac patients with positive expectations about their chance of recovery and return to a normal lifestyle 
are believed to enjoy better long-term survival together with better functional status. Various potential clinical, demographic, and psychosocial variables do not contribute to creating these effects (6). Patient and family also act as a barrier to behavioral change in two ways: our study participants expressed their uncertainty about accomplishing their task in cardiac rehabilitation sessions; this negative judgment about their own ability acted as an obstacle to their engagement. Based on the research results, self-efficacy is a key element in heart disease self-management programs. The interdisciplinary nature of the program and the design of the framework of behavioral change theories result in patient-centered programs (23). Previous research has demonstrated the importance of family support in the recovery of cardiac patients. In contrast, our study population cited family overprotection as a hindrance to their ability. Cultural diversity is another variable of significance that merits due attention. For all cultures that accord great value to individualism and autonomy, there are those that attach great import to interdependence (24). In the context of Iran, the extended family underpins the individual's societal role. Consequently, the whole family feels obliged to provide care for its members in sickness. Nonetheless, the degree and type of the involvement of the family in the care of patients are largely unknown. Also, patients with chronic illnesses do not always experience family involvement positively. Chronic disease patients receiving family care often complain about being criticized and nagged at and, as such, are made to feel guilty about the care (25). The behavioral change challenges originating from the interaction between our study subjects' values system and cognitive system are illustrated in Figure 1.

The importance of modifiable risk factors for the prevention of premature mortality in cardiac patients is widely recognized by researchers and public health professionals. Our results suggest that during the cardiac rehabilitation program, our study population's behavioral change challenges acted at two levels: interpersonal and intrapersonal. At intrapersonal level, factors such as value, knowledge, attitude about heart disease, self-efficacy to fulfill the rehabilitation task, and family overprotection were the main issues that emerged. Therefore, it is advisable that culturally-based interventions be formulated to bolster disease self-management.

\section{Acknowledgements}

This study was part of the $\mathrm{PhD}$ thesis of the first author, conducted in cardiac rehabilitation centers affiliated with Isfahan University of Medical Sciences. The authors would like to thank all the participants, who made this study possible.

\section{Funding/Support}

This research was financially supported by Isfahan University of Medical Sciences, Isfahan, Iran.

\section{References}

1. Drozd F, Lehto T, Oinas-Kukkonen H. Persuasive Technology. Design for Health and Safety. Springer; 2012. pp. 157-68.Exploring perceived persuasiveness of a behavior change support system: a structural model.

2. Piepoli MF, Corra U, Benzer W, Bjarnason-Wehrens B, Dendale P, Gaita D, et al. Secondary prevention through cardiac rehabilitation: from knowledge to implementation. A position paper from the Cardiac Rehabilitation Section of the European Association of Cardiovascular Prevention and Rehabilitation. EurJCardiovasc Prev Rehabil. 2010;17(1):1-17.

3. Bjarnason-Wehrens B, McGee H, Zwisler AD, Piepoli MF, Benzer W, Schmid JP, et al. Cardiac rehabilitation in Europe: results from the European Cardiac Rehabilitation Inventory Survey. Eur J Cardiovasc Prev Rehabil. 2010;17(4):410-8.

4. Fisher EB, Fitzgibbon ML, Glasgow RE, Haire-Joshu D, Hayman LL, Kaplan RM, et al. Behavior matters. Am J Prev Med. 2011; 40(5):e15-30.

5. Michie S, van Stralen MM, West R. The behaviour change wheel:a new method for characterising and designing behaviour change interventions. Implement Sci. 2011;6:42.

6. Barefoot JC, Brummett BH, Williams RB, Siegler IC, Helms MJ, Boyle $\mathrm{SH}$, et al. Recovery expectations and long-term prognosis of patients with coronary heart disease. Arch Intern Med. 2011;171(10):929-35.

7. Newsom JT, Huguet N, McCarthy MJ, Ramage-Morin P, Kaplan MS, Bernier J, et al. Health behavior change following chronic illness in middle and later life. J Gerontol B Psychol Sci Soc Sci. 2012;67(3):279-88.

8. Schwarzer R. Modeling health behavior change: How to predict and modify the adoption and maintenance of health behaviors. Appl Psychol. 2008;57(1):1-29.

9. Huang ES, Basu A, O'Grady M, Capretta JC. Projecting the future diabetes population size and related costs for the U.S. Diabetes Care. 2009;32(12):2225-9.

10. Peterson JC, Allegrante JP, Pirraglia PA, Robbins L, Lane KP, Boschert KA, et al. Living with heart disease after angioplasty: A qualitative study of patients who have been successful or unsuccessful in multiple behavior change. Heart Lung. 2010; 39(2):105-15.

11. O'Neil A, Berk M, Davis J, Stafford L. Cardiac-self efficacy predicts adverse outcomes in coronary artery disease (CAD) patients. Health. 2013;5(6).

12. Patton MQ. Qualitative evaluation and research methods.: SAGE Publications; 1990.

13. Rodriguez KL, Appelt CJ, Switzer GE, Sonel AF, Arnold RM. "They diagnosed bad heart": a qualitative exploration of patients' knowledge about and experiences with heart failure. Heart Lung. 2008;37(4):257-65.

14. Braun V, Clarke V. Using thematic analysis in psychology. Qual Res Psychol. 2006;3(2):77-101.

15. Major B, O'Brien LT. The social psychology of stigma. Annu Rev Psychol. 2005;56:393-421.

16. Wrigley S, Jackson H, Judd F, Komiti A. Role of stigma and attitudes toward help-seeking from a general practitioner for mental health problems in a rural town. Aust N Z J Psychiatry. 2005;39(6):514-21.

17. Lubkin IM, Larsen PD. Chronic illness: Impact and interventions.: Jones \& Bartlett Learning; 2006.

18. Osborn CY, Paasche-Orlow MK, Bailey SC, Wolf MS. The mechanisms linking health literacy to behavior and health status. Am J Health Behav. 2011;35(1):118-28.

19. Ford D, Zapka J, Gebregziabher M, Yang C, Sterba K. Factors associated with illness perception among critically ill patients and surrogates. CHEST J. 2010;138(1):59-67.

20. Greco A, Steca P, Pozzi R, Monzani D, Malfatto G, Parati G. The influence of illness severity on health satisfaction in patients with cardiovascular disease: The mediating role of illness perception and self-efficacy beliefs. Behav Med. 2015;41(1):9-17.

21. Petrie KJ, Weinman J. Patients' perceptions of their illness the dynamo of volition in health care. Curr Dir Psychol Sci. 2012; 21(1):60-5. 


\section{JokarFetal.}

22. Saarni SI, Suvisaari J, Sintonen H, Pirkola S, Koskinen S, Aromaa A, et al. Impact of psychiatric disorders on health-related quality of life: general population survey. BrJPsychiatry. 2007;190:326-32.

23. Katch $\mathrm{H}$, Mead $\mathrm{H}$. The role of self-efficacy in cardiovascular dis ease self-management: a review of effective programs. Patient Intell. 2010;2:33-44.
24. Kagawa-Singer M, Padilla GV, Ashing-Giwa K. Health-related quality of life and culture. Semin Oncol Nurs. 2010;26(1):59-67.

25. Rosland AM, Heisler M, Choi HJ, Silveira MJ, Piette JD. Family influences on self-management among functionally independent adults with diabetes or heart failure: do family members hinder as much as they help? Chronic Illn. 2010;6(1):22-33. 